\title{
Establishment and Assessment of An Amplicon Sequencing Method Targeting The 16S-ITS-23S rRNA Operon For Analysis of The Equine Gut Microbiome
}

Yuta Kinoshita ( $\nabla$ kinoshita@equinst.go.jp )

Japan Racing Association

Hidekazu NIWA

Japan Racing Association

Eri UCHIDA-FUJII

Japan Racing Association

Toshio NUKADA

Japan Racing Association

\section{Research Article}

Keywords: Microbial communities, rRNA, CCMetagen pipeline, archaeal microbiota

Posted Date: February 2nd, 2021

DOl: https://doi.org/10.21203/rs.3.rs-156589/v1

License: (c) (i) This work is licensed under a Creative Commons Attribution 4.0 International License.

Read Full License 


\section{Abstract}

Microbial communities are commonly studied by using amplicon sequencing of part of the 16S rRNA gene. Sequencing of the full-length $16 \mathrm{~S}$ rRNA gene can provide higher taxonomic resolution and accuracy. To obtain even higher taxonomic resolution, with as few false-positives as possible, we assessed a method using long amplicon sequencing targeting the rRNA operon combined with a CCMetagen pipeline. Taxonomic assignment had $>90 \%$ accuracy at the species level in a mock sample and at the family level in equine fecal samples, generating similar taxonomic composition as shotgun sequencing. The rRNA operon amplicon sequencing of equine fecal samples underestimated compositional percentages of bacterial strains containing unlinked rRNA genes by a third to almost a half, but unlinked rRNA genes had a limited effect on the overall results. The rRNA operon amplicon sequencing with the A519F + U2428R primer set was able to reflect archaeal genomes, whereas fulllength 16S rRNA with $27 \mathrm{~F}+1492 \mathrm{R}$ could not. Therefore, we conclude that amplicon sequencing targeting the rRNA operon captures more detailed variations of bacterial and archaeal microbiota.

\section{Introduction}

The horse is a hindgut fermenter, and most microbial activities in the gastrointestinal (GI) tract take place in the large intestine, so the majority of intestinal microbes reside in the colon and the comparatively enlarged caecum ${ }^{1}$. The degradation of indigestible cellulosic and hemi-cellulosic forage components by these microbes is crucial for the bioavailability of energy and essential nutrients in horses ${ }^{2}$. Studies of these activities in the GI tract of horses have focused on bacteria, but fungi and archaea are also involved ${ }^{3,4}$. In particular, methanogenic archaea, widely found in the equine hindgut, metabolize $\mathrm{H}_{2}$ and $\mathrm{CO}_{2}$ to methane, and are considered to support the carbohydrate-degrading activity of cellulolytic bacteria in the hindgut ${ }^{4-6}$. The GI microbiota also plays a crucial role in maintaining normal microbial flora by various methods: it produces antimicrobial products, competes directly for nutrients with pathogens, inhibits or inactivates bacterial toxins, and produces bacteriocins and short-chain fatty acids, which inhibit the growth of pathogens and pathobionts ${ }^{7}$. It has also been shown to modify virulence factor expression of pathogens and to facilitate the host barrier function through the upregulation of mucus production, antimicrobial molecules, and secretion of $\lg \mathrm{A}^{7}$.

High-throughput sequencing technologies are widely used to investigate microbial flora in many environments, such as oral, intestinal, vaginal, aquatic, and soil. Amplicon sequencing targeting a specific region in microbial genomes is one methodology that uses high-throughput sequencing, and a partial region of the 16S rRNA gene is commonly used to study microbiomes ${ }^{8}$. The third generation of sequencing technologies now allows the production of very long reads, albeit with higher error rates, in real time ${ }^{9,10}$. Yet despite the higher error rates, analysis of the full-length $16 \mathrm{~S}$ rRNA gene amplicon by nanopore sequencing technology has a comparable or higher taxonomic resolution of bacterial flora at the genus and particularly species levels than short-read sequencing on an Illumina platform ${ }^{11-13}$. Long amplicon analysis targeting the nearly complete 16S-ITS-23S rRNA operon has been used to achieve 
greater confidence in taxonomic assignment ${ }^{14-16}$, but it could miss some proportion of bacterial species on account of unlinked rRNA genes. Unlinked rRNA genes are widespread among bacteria and archaea: at rates of $13 \%-41 \%$ in soil, $7.7 \%-29 \%$ in sediment, and $8.1 \%-8.8 \%$ in anaerobic digesters, though not in the human gut ${ }^{17}$. In addition, the incompleteness of databases for the 16S-ITS-23S rRNA operon could be a limitation in using the operon region in metagenome sequencing; i.e., some false-positives could be assigned owing to an insufficient record of bacterial and archaeal species or the short sequence lengths of the deposited microbes ${ }^{14,16}$.

Our main aim in this study was to reveal the features and limitations of rRNA operon amplicon sequencing in metagenomic analysis of equine fecal samples in comparison with full-length 16S rRNA gene sequencing and shotgun sequencing. First, we evaluated the two amplicon sequencing methods with different PCR polymerase kits and reference databases for taxonomic assignments, using a mock bacterial community. Second, we explored unlinked rRNA genes in equine feces and assessed their influence on overall taxonomic compositional results generated by the rRNA operon amplicon sequencing. Finally, we compared the results to reveal the advantages and disadvantages of each sequencing method.

\section{Materials And Methods}

\section{Genomic DNA of mock bacterial community}

A bacterial DNA cocktail of a mock community (DNA-Mock-001) was obtained from the National Institute

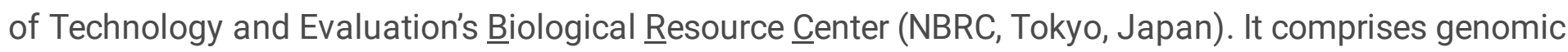
DNA prepared from 15 bacterial strains (Table S4).

\section{Fecal sampling and DNA extraction}

Fecal samples were collected from three female Thoroughbred horses (Equus ferus caballus): A and S (2 years old) and $L$ (9 years old). All horses were clinically healthy, with no apparent history of intestinal problems. No antimicrobials had been administered to them for at least a month before sampling. The samples from horses $A$ and $S$ were immediately sampled just after being dropped on the straw bed in the stable. The sample from horse $L$ was collected directly from the rectum. The samples were immediately placed on ice and the genomic DNA of each was extracted within $1 \mathrm{~h}$ after collection. For DNA extraction, $3 \mathrm{~g}$ of wet fecal sample was suspended vigorously in $40 \mathrm{~mL}$ of PBS and then left for $1 \mathrm{~min}$ at room temperature to remove large plant debris. Then $4 \mathrm{~mL}$ of the suspension was centrifuged at $13000 \times \mathrm{g}$ for $2 \mathrm{~min}$ at $4{ }^{\circ} \mathrm{C}$, and the pellet was washed twice with TE buffer containing $10 \mathrm{mM}$ Tris and $1 \mathrm{mM}$ EDTA. The final pellet was suspended in $800 \mu \mathrm{L}$ of distilled water. The genomic DNA was extracted from the suspension by using a Quick-DNA Fecal/Soil Microbe Kit (Zymo Research, Tokyo, Japan) following the manufacturer's instructions. It was purified with an Agencourt AMPure XP purifier (Beckman Coulter, Brea, CA, USA), and short DNA ( $<10 \mathrm{~kb}$ ) was depleted by using a short-read eliminator (XS; Circulomics, Baltimore, MD, USA). 
All samplings and experiments were conducted in accordance with ethical and welfare regulations of the Animal care committee of the Equine Research Institute. The Animal care committee approved all experimental protocols. We also complied with the ARRIVE guidelines (https://arriveguidelines.org/).

\section{Sequencing and base-calling for mock community}

Genomic libraries for two target regions (full-length 16S rRNA gene and 16S-ITS-23S rRNA operon) were prepared by using the four-primer PCR method protocol, version FFP_9038_v108_revN_14Aug2019 (Oxford Nanopore Technologies [ONT], Oxford, UK) with slight modifications. The four-primer PCR uses two target-specific inner primers with a 5 ' tail and two universal outer primers which prime off the tail on the $5^{\prime}$ end of the inner primers (Table S5), resulting in the generation of target amplicons with barcodes ${ }^{15,32,36}$. PCR amplifications were conducted using either LongAmp ${ }^{\mathrm{TM}}$ Taq $2 \times$ Master Mix (New England Biolabs, Ipswich, MA, USA) or the KAPA2G ${ }^{\mathrm{TM}}$ Robust HotStart Ready Mix PCR Kit (Kapa Biosystems, Wilmington, MA, USA). PCR was performed in a total volume of $25 \mu \mathrm{L}$ containing the inner primers (50 $\mathrm{nM}$ each), the barcoded outer primer mixture (300 nM) from the PCR barcoding kit (SQKPBK004; ONT), and the DNA cocktail (1 ng) as template. PCR conditions are shown in Table S6. The PCR amplicons were purified with the Agencourt AMPure XP purifier and quantified by a NanoDrop spectrophotometer (ThermoFisher Scientific), and the libraries were sequenced on a MinION sequencer using R9.4.1 flow cells (FLO-MIN106D; ONT) following the manufacturer's instructions. Base-calling of raw fast5 data from the MinION was carried out in Guppy v. 3.6.1 software (ONT) with its

"--trim_barcodes" option for removing sequencing adapters and barcodes. Details on each sample, including number of reads, median read lengths, and median read quality, are shown in Table S7.

\section{Sequencing and base-calling for fecal samples}

For fecal samples, both amplicon sequencing and shotgun sequencing were conducted. Genomic libraries for amplicon sequencing targeting the full-length 16S rRNA gene or the 16S-ITS-23S rRNA operon were prepared as for the mock community by using a KAPA2G Robust HotStart Ready Mix PCR Kit. Genomic libraries for shotgun sequencing were prepared by using a Rapid Barcoding Kit (SQK-RBK004; ONT) following the manufacturer's instructions. All libraries were sequenced on the MinION sequencer using R9.4.1 flow cells. Base-calling of raw fast 5 data was carried out as above; details are shown in Table S7.

\section{Sequencing data processing for mock community}

The raw FASTQ files were pretrimmed in Seqkit v. 0.12 .0 software $^{37}$ to filter by quality scores of 10 , with lengths of $1,300 \mathrm{bp}$ for the full-length 16S rRNA gene and 3,500 bp for the 16S-ITS-23S rRNA operon. Afterward, 30,000 reads were subsampled in Seqtk v. 1.3. software (https://github.com/lh3/seqtk), and chimera reads were removed in yacrd v. 0.6.1 software ${ }^{38}$. Twenty thousand quality-controlled reads were selected by quality score $>12$ and by size: $1,300-1,950$ bp for the full-length $16 \mathrm{~S}$ rRNA gene and 3,500$5,000 \mathrm{bp}$ for the 16S-ITS-23S rRNA operon. Accurate taxonomic assignments of the quality-controlled 
read sets were performed with the CCMetagen pipeline by coupling with $\mathrm{KMA}^{19}$ and CCMetagen ${ }^{20}$ software, i.e., read mapping to a reference database in KMA software (-mem_mode, -bcNano, and -1t1 options), specifying the minimum phred score (-mp 20), minimum alignment score (-mrs 0.0$)$, and basecalling option (-bc 0.7 ), followed by a quality-filtering step in CCMetagen software with default settings. Two reference databases-rrn DB ${ }^{18}$ and ncbi_202006 DB, described below-were used in the taxonomic assignment step. The dissimilarity indices between the percentage of each sequencing condition excluding mis- or unidentified values and that of the expected abundance of the mock community were calculated by the vegan package in $\mathrm{R}$ v. 3.6.1 software with default settings ${ }^{39,40}$.

\section{Sequencing data processing for fecal samples}

For the FASTQ files from the amplicon sequencing, pretrimmed reads were created in Seqkit software to filter by quality scores of 10 , with lengths of $1,300 \mathrm{bp}$ for the full-length $16 \mathrm{~S}$ rRNA gene and 3,500 bp for the 16S-ITS-23S rRNA operon. Chimera reads were removed in yacrd software. Quality-controlled reads were selected by quality score $>11$ and by size: 1,300-1,950 bp for the full-length $16 \mathrm{~S}$ rRNA gene and 3,500-5,000 bp for the 16S-ITS-23S rRNA operon. Taxonomic assignments were performed as above.

For the FASTQ files from the shotgun sequencing, quality-controlled data sets were created in Seqkit software to filter by lengths of $1,000 \mathrm{bp}$ and quality scores of 10 . After conversion from FASTQ format to FASTA format, rRNA genes were detected in each sequence read in Barrnap v. 0.9 software (https://github.com/tseemann/barrnap) with default settings, and the reads which contained rRNA genes were extracted from the raw FASTA files in Seqkit software: 2,333 sequence reads from horse A, 4,417 reads from horse $S$, and 8,700 reads from horse $L$. The taxon assignments of each read against ncbi_202006 DB were performed as above. For further investigations of unlinked rRNA genes, the reads that could be classified to at least phylum level were kept, and we classified reads as containing unlinked rRNA genes following the previous criteria ${ }^{17}$, namely if there was $>1,500$ bp between the $16 \mathrm{~S}$ and $23 \mathrm{~S}$ rRNA genes, or if there was no $23 \mathrm{~S}$ domain found by $1,500 \mathrm{bp}$ after the end of the $16 \mathrm{~S}$ rRNA. We removed the sequence reads for final analyses that could not be judged to contain linked or unlinked rRNA genes (i.e., with $<1,500 \mathrm{bp}$ after the 3 ' end of the $16 \mathrm{~S}$ rRNA gene), and we kept only reads that included a $16 \mathrm{~S}$ rRNA gene to avoid potential double-counting organisms with unlinked 16S and 23S rRNA genes. Information on all long-read sequences included is shown in Table S1.

The ggplot2 package in R v. 3.6.1 software was used to depict the percentages of quality-filtered reads from the CCMetagen pipeline in each taxon and boxplots for the top 20 orders with the highest average percentage ${ }^{41}$. The NMDS analysis was carried out using the vegan package in $\mathrm{R}$ software with default settings ${ }^{39}$. A heat map showing the percentage of each order identified was plotted in ComplexHeatmap software in R, specifying the distance measure "spearman" and clustering method "ward.D2" for hierarchical clustering ${ }^{42}$.

\section{Taxonomic reference databases}


We used two databases which contain sequences of the 16S-ITS-23S rRNA operon: (1) rrn DB, which includes sequences from the whole ribosomal operon of 22,351 bacterial species retrieved from GenBank $^{18}$, and (2) ncbi_202006 DB, which was created in this study. To create ncbi_202006 DB, all of the bacterial and archaeal data were downloaded from NCBI in June 2020 in genome_updater v. 0.2.2 software (https://github.com/pirovc/genome_updater), and rRNA genes were predicted and extracted in Barrnap software. Each rRNA gene in the same nucleotide sequence was concatenated if the distance between rRNA genes was $<2,000 \mathrm{bp}$, because the lengths of ITS regions in linked rRNA genes were by definition $<1,500 \mathrm{bp}^{17}$. All sequences whose lengths were $<1,000 \mathrm{bp}$ were removed from the database. The final ncbi_20206 DB contains 493,329 sequences. Accessions for ncbi_20206 DB are available at https://bitbucket.org/ykinoshita1984/ncbi_db/downloads/.

\section{Statistical analysis}

The vegan package in $\mathrm{R}$ software was used to conduct permutational multivariate analysis of variance (PERMANOVA), specifying the Bray-Curtis method and 10,000 permutations ${ }^{39} . P<0.05$ was considered to indicate a significant difference.

\section{Results And Discussion}

\section{Mock community}

Comparisons of metagenomic sequence data acquired with several combinations of conditions from defined mock communities are important for the assessment of sequencing platform performance and downstream analyses, including taxonomic assignment. Using a commercial mock community containing DNAs of 15 bacteria, we compared metagenomic sequences from three sources: (1) PCR polymerase kits-LongAmp ${ }^{\mathrm{TM}}$ Taq 2x Master Mix and KAPA2G ${ }^{\mathrm{TM}}$ Robust HotStart Ready Mix PCR Kit; (2) PCR target regions-full-length 16S rRNA gene ( 1450 bp) and 16S-ITS-23S rRNA operon ( 4000 bp); and (3) reference databases -the $r r n$ operon database, rrn DB $^{18}$, and the ncbi_202006 DB, newly designed in this study. The degree of assignment of 20,000 quality-controlled reads at the genus or species level is shown in Fig. 1. Among the eight sequencing conditions at genus level, the lowest values of both dissimilarity index with the expected abundance of the mock community (0.21) and the percentage of "mis- or unidentified" (1.3\%) were observed in a combination of KAPA2G ${ }^{T M}$, rRNA operon, and ncbi_202006 DB. At the species level, the same combination obtained the lowest values (0.21 of dissimilarity index and $6.2 \%$ of "mis- or unidentified"). Their beta diversities were explained mainly by PCR target region (genus level, $R^{2}=0.51946, P=0.0042$; species level, $R^{2}=0.55341, P=0.0036$, PERMANOVA, Table 1). The PCR polymerase kits and reference databases had less effect on the taxonomic assignment than the PCR target regions, but still affected both the dissimilarity from the expected abundance of the mock community and the percentage "mis- or unidentified". In total, the combination of KAPA2G, rRNA operon, and ncbi_202006 DB performed best at assigning the genus and particularly the species in terms of both dissimilarity and percentage "mis- or unidentified". Therefore, we compared shotgun sequencing method and two amplicon sequencing methods in the following 
experiments: KAPA2G + rRNA operon + ncbi_202006 DB and KAPA2G + full-length 16S rRNA gene + ncbi_202006 DB.

\section{Unlinked rRNA genes}

Unlinked rRNA genes are widespread in natural environments such as soil but have not been detected in the human gut ${ }^{17}$. The presence or absence of unlinked rRNA genes in the gut of other animals has been unclear. Here, we found sequence reads of unlinked rRNA genes in all three equine fecal samples (Tables 2, S1). The feces from horses $A$ and $S$ were collected immediately after they had fallen on the straw bed, so contamination with unlinked rRNA genes from the straw bed cannot be completely ruled out. The feces from horse L, however, was collected directly from the rectum, with no opportunity for contamination. All reads with unlinked rRNA genes found from the horses were mapped in KMA v. 1.3.0 software ${ }^{19}$ to genus Cellulosilyticum or Anaerocolumna and were quality filtered by CCMetagen v. 1.2.2 software ${ }^{20}$ to family Lachnospiraceae, order Clostridiales, or phylum Firmicutes, depending on their mapping qualities. Members of the Lachnospiraceae can ferment diverse plant polysaccharides to produce short-chain fatty acids ${ }^{21}$, and make up the largest single group in rumen microbes of cows ${ }^{22}$. No unlinked rRNA in three complete genomes of genus Anaerocolumna was found (Table S2). The complete genome of Anaerocolumna aminovalerica, which was mapped by a sequence read in this study, was not registered in the National Center for Biotechnology Information (NCBI) database as at the end of 2020, and the presence of unlinked rRNA genes in Anaerocolumna remains to be studied. On the other hand, we found that the genome of Cellulosilyticum lentocellum DSM 5427 has multiple rRNA copies with a combination of linked- and unlinked rRNA genes, being defined as "mixed type"17,23 (Table S2). We confirmed that Cellulosilyticum sp. WCF-2 (accession no. NZ_CP034675), which was isolated from cow feces, also has unlinked rRNA genes. Therefore, we believe that the equine gut microbiota, unlike that in the human gut, contains unlinked rRNA genes, and that other herbivores-at least cows-also might have unlinked rRNA genes in the gut microbiota. The average length of the ITS regions in unlinked rRNA genes was almost 410,000 bp ${ }^{17}$, and could not be PCR-amplified before sequencing because of its long length. So rRNA operon amplicon sequencing might underestimate the occurrence of these strains with unlinked rRNA genes. We evaluated the degree of underestimation next.

\section{CCMetagen pipeline and Healthy fecal samples}

Clustering of operational taxonomic units (OTUs) and amplicon sequence variant analysis are commonly conducted for $16 \mathrm{~S}$ amplicon sequencing on the Illumina platform. These steps can reduce duplications and errors of representative sequences, resulting in a more reliable taxonomic assignment ${ }^{24,25}$. But there are no solid consensus methods for OTU clustering or amplicon sequence variant analysis to obtain accurate taxonomic assignment in nanopore sequencing, and the latter's higher error rate could overestimate the bacterial diversity in samples ${ }^{16,26}$. To avoid overestimation, several methods have been used with nanopore data: e.g., reconstructing the consensus sequence ${ }^{16}$ or removing singleton reads or OTUs, or removing reads or OTUs whose percentages are less than arbitrary values such as $0.005 \% 12,27$. 
In addition, the taxonomic identification of metagenomic reads is generally more reliable at higher taxonomic levels (e.g., phylum or class) than at lower levels (e.g., genus or species), but it is not easy to decide which taxonomic level is reliable enough, because it varies by metagenomic read. Here, we used a CCMetagen pipeline to obtain accurate taxonomic assignments at appropriate taxonomic levels by conducting quality-filtering steps in CCMetagen v. 1.2.2 software ${ }^{20}$ after the read mappings in KMA software. This pipeline reportedly outperforms current analytic methods (i.e., Kraken2, Centrifuge, and KrakenUniq) in making accurate taxonomic classifications ${ }^{20}$. Although comparisons with other assignment methods to apply to nanopore reads remain to be made, the CCMetagen pipeline revealed that the average percentages of taxonomic identification at phylum, class, and order levels in fecal samples were $>90 \%$ by all three sequencing sources (Fig. 2). In addition, the average percentages at the family level by the two amplicons were also high enough to use for downstream analysis: $96.1 \%$ by $16 \mathrm{~S}$ rRNA sequencing and $98.8 \%$ by rRNA operon sequencing. The percentages of taxa identified dropped sharply at genus level to $24.1 \%$ by 16 S rRNA sequencing and $46.9 \%$ by rRNA operon sequencing, while those for the mock community were still comparatively high at genus level (93.3\% and $98.7 \%$, respectively) (Figs. 1,2). These results might be attributed to the compositional complexities of the fecal samples and deficits of registered genome information in the public databases of relevance to the equine gut microbiota. The results at the order level, which are the lowest among the taxa with a taxonomic identification rate of $\geq 90 \%$ in all sequencing methods, were used for downstream analysis. Depiction of the numbers of orders detected against the numbers of reads analyzed showed that the curve started to plateau at around 30,000 reads (Fig. 3). So we randomly subsampled 30,000 reads from each combination of analytical conditions and used them in further experiments to analyze the composition of the equine gut microbiota.

We first compared the three sequencing methods in terms of the unlinked rRNA genes. The percentages of genus Cellulosilyticum which had unlinked rRNA genes are shown in Table S3. The percentages ranged from $0.59 \%$ to $1.54 \%$ in horse $A, 0.34 \%$ to $0.98 \%$ in horse $S$, and $0.14 \%$ to $0.73 \%$ in horse $L$, showing that the values differed slightly depending on the sequencing method. The average value by rRNA operon amplicon sequencing $(0.36 \%)$ was roughly a third to a half of the percentages by the other two methods ( $0.85 \%$ by $16 \mathrm{~S}$ rRNA amplicon sequencing, $1.02 \%$ by shotgun sequencing). These results showed that bacterial strains with unlinked rRNA genes could account for approximately $1 \%$ of the equine fecal microbiota and amplicon sequencing targeting the rRNA operon underestimated the percentages of bacterial species with unlinked rRNA genes.

To evaluate the influence of the underestimation of unlinked rRNA genes on the overall metagenomic results, we performed non-metric multidimensional scaling (NMDS) analysis. The taxonomic groups of the metagenomes generated from the same sequencing method were significantly similar (PERMANOVA: $P=0.0037, R^{2}=0.74328$ ), but the individual horse did not have a significant effect (PERMANOVA: $P=$ $0.6553, R^{2}=0.07468$ ). The analysis showed clear differences among the three sequencing methods (Fig. 4). Despite the influence of the unlinked rRNA genes, the plots of the rRNA operon were distributed between those of the other methods, not generating an obvious independent group as compared to the 
other sequencings. In addition, clustering analysis shown by heat map grouped the compositions of the rRNA operon with those from the shotgun sequencing, showing that rRNA operon amplicon sequencing did not make an independent group by itself (Fig. 5). Overall, we found that amplicon sequencing targeting the rRNA operon could underestimate the percentage of bacterial strains containing unlinked rRNA genes by a third to almost half when applied to equine fecal samples, but the effects on the overall metagenomic results were limited, and the operon targeted sequencing could generate similar metagenomic results to those by shotgun sequencing, which has no PCR bias.

We further explored differences between the three sequencing methods. Orders Clostridiales and Lactobacillales were dominant in all fecal samples by all methods (Figs. 5, 6). The averages of Orders Clostridiales and Lactobacillales were $67.7 \%$ and $15.8 \%$ in 16 S rRNA gene sequencing, $48.9 \%$ and $30.7 \%$ in rRNA operon sequencing, and $40.3 \%$ and $40.2 \%$ in shotgun sequencing, respectively. These results are compatible with previous reports that the Firmicutes, which encompass the Clostridiales and the Lactobacillales, was the most dominant taxon in fecal samples from healthy Thoroughbreds ${ }^{28,29}$. Other taxa were much less predominant, but several taxa from each sequencing method had considerably lower abundances than those from the other sequencing methods: e.g., Desulfovibrionales and Corynebacteriales in 16S rRNA sequencing, Spirochaetales and Campylobacterales in rRNA operon sequencing, and Acidaminococcales in shotgun sequencing (Fig. 6). Several factors are known to affect taxonomic assignment, including primer sets, PCR conditions, and reference databases ${ }^{30-33}$. We used the same database, ncbi_202006 DB, in all the three sequencing methods, and attribute the differences described above mainly to primer mismatch or to PCR conditions such as annealing temperature. The most notable and interesting differences among the three sequencing methods were found in the archaea, in the orders Methanobacteriales and Methanomicrobiales. While 16S rRNA gene sequencing with the $27 \mathrm{~F}+1492 \mathrm{R}$ primer set could not find any archaeal genomes, both rRNA operon sequencing and shotgun sequencing could detect archaea, at mean values of 3.5\% (1.9\% of Methanobacteriales and 1.6\% of Methanomicrobiales) and 3.3\% (3.0\% and 0.3\%), respectively (Fig. 6). These archaea produce methane from $\mathrm{H}_{2}$ and $\mathrm{CO}_{2}$ and might boost the carbohydrate-degrading activity of cellulolytic bacteria ${ }^{4,6}$; therefore, methanogenic archaea should be more intensively focused on to unveil the mechanisms and functional interactions among microbes needed for successful degradation of nutrients. The diversity and importance of the archaea in various environments remain poorly understood ${ }^{34}$; one reason is that few primer sets have been validated ${ }^{35}$. The primer set used for the rRNA operon amplicon sequencing in this study-A519F + U2428R-covers a large proportion of the known bacterial and archaeal rRNA genes ${ }^{15}$, and the ratio of archaea is comparable to the results obtained by shotgun sequencing, showing that amplicon sequencing using primers A519F + U2428R to amplify the rRNA operon could be a powerful tool to investigate microbiota, including archaea.

\section{Conclusions}

We compared amplicon sequencing targeting the rRNA operon with the A519F + U2428R primer set with sequencing targeting the full-length $16 \mathrm{~S}$ rRNA gene with the $27 \mathrm{~F}+1492 \mathrm{R}$ set and shotgun sequencing. 
Even though 16S rRNA gene amplicon sequencing has low taxonomic resolution at the genus and especially species levels and cannot evaluate archaeal genomes, the full-length 16S rRNA gene is still a promising target for metagenomic analysis using long amplicons because of its robustness among bacterial communities and the availability of comprehensive databases. rRNA operon amplicon sequencing combined with the KAPA2G ${ }^{\mathrm{TM}}$ Robust HotStart Ready Mix PCR Kit and the ncbi_202006 DB, a newly designed reference database, could offer confident taxonomic assignments even at lower ranks. Unlike the 16S rRNA gene method, the rRNA operon method can reflect both bacterial and archaeal microbiota in equine feces, so researchers can capture fecal microbiota in more detail in horse.

\section{Declarations}

\section{Data availability}

All nanopore sequencing data generated in this study are available under the BioProject accession number PRJDB10841, with BioSamples accession numbers SAMD00260421 (mock community), SAMD00260422 (horse A), SAMD00260423 (horse S), and SAMD00260424 (horse L).

\section{Acknowledgements}

This study was supported by the Japan Racing Association, which provided all help in the design of the study, the collection, analysis, and interpretation of data, and writing the manuscript.

\section{Author contributions}

Y.K. did experimental works and wrote the main manuscript. H.N., E.U.F, and T.N. reviewed the manuscript.

\section{Competing interests}

The authors declare no competing interests.

\section{References}

1. Blackmore, T. M. et al. Strong stability and host specific bacterial community in faeces of ponies. PLoS One 8, e75079, doi:10.1371/journal.pone.0075079 (2013).

2. Julliand, V. \& Grimm, P. HORSE SPECIES SYMPOSIUM: The microbiome of the horse hindgut: History and current knowledge. J Anim Sci 94, 2262-2274, doi:10.2527/jas.2015-0198 (2016).

3. Moore, B. E. \& Dehority, B. A. Effects of diet and hindgut defaunation on diet digestibility and microbial concentrations in the cecum and colon of the horse. J Anim Sci 71, 3350-3358, doi:10.2527/1993.71123350x (1993).

4. Jensen, B. B. Methanogenesis in monogastric animals. Environ Monit Assess 42, 99-112, doi:10.1007/BF00394044 (1996). 
5. Dougal, K. et al. A comparison of the microbiome and the metabolome of different regions of the equine hindgut. FEMS Microbiol Ecol 82, 642-652, doi:10.1111/j.1574-6941.2012.01441.x (2012).

6. Flint, H. J., Scott, K. P., Duncan, S. H., Louis, P. \& Forano, E. Microbial degradation of complex carbohydrates in the gut. Gut Microbes 3, 289-306, doi:10.4161/gmic.19897 (2012).

7. Kamada, N., Chen, G. Y., Inohara, N. \& Nunez, G. Control of pathogens and pathobionts by the gut microbiota. Nat Immunol 14, 685-690, doi:10.1038/ni.2608 (2013).

8. Ranjan, R., Rani, A., Metwally, A., McGee, H. S. \& Perkins, D. L. Analysis of the microbiome: Advantages of whole genome shotgun versus $16 \mathrm{~S}$ amplicon sequencing. Biochem Biophys Res Commun 469, 967-977, doi:10.1016/j.bbrc.2015.12.083 (2016).

9. Jain, M., Olsen, H. E., Paten, B. \& Akeson, M. The Oxford Nanopore MinION: delivery of nanopore sequencing to the genomics community. Genome Bio/17, 239, doi:10.1186/s13059-016-1103-0 (2016).

10. Eid, J. et al. Real-time DNA sequencing from single polymerase molecules. Science 323, 133-138, doi:10.1126/science.1162986 (2009).

11. Shin, J. et al. Analysis of the mouse gut microbiome using full-length 16S rRNA amplicon sequencing. Sci Rep 6, 29681, doi:10.1038/srep29681 (2016).

12. Nygaard, A. B., Tunsjo, H. S., Meisal, R. \& Charnock, C. A preliminary study on the potential of Nanopore MinION and Illumina MiSeq 16S rRNA gene sequencing to characterize building-dust microbiomes. Sci Rep 10, 3209, doi:10.1038/s41598-020-59771-0 (2020).

13. Heikema, A. P. et al. Comparison of Illumina versus Nanopore $16 \mathrm{~S}$ rRNA Gene Sequencing of the Human Nasal Microbiota. Genes (Basel) 11, doi:10.3390/genes11091105 (2020).

14. Cusco, A., Catozzi, C., Vines, J., Sanchez, A. \& Francino, O. Microbiota profiling with long amplicons using Nanopore sequencing: full-length 16S rRNA gene and the 16S-ITS-23S of the rrn operon. F1000Res 7, 1755, doi:10.12688/f1000research.16817.2 (2018).

15. Martijn, J. et al. Confident phylogenetic identification of uncultured prokaryotes through long read amplicon sequencing of the 16S-ITS-23S rRNA operon. Environ Microbio/ 21, 2485-2498, doi:10.1111/1462-2920.14636 (2019).

16. Kerkhof, L. J., Dillon, K. P., Haggblom, M. M. \& McGuinness, L. R. Profiling bacterial communities by MinION sequencing of ribosomal operons. Microbiome 5, 116, doi:10.1186/s40168-017-0336-9 (2017).

17. Brewer, T. E. et al. Unlinked rRNA genes are widespread among bacteria and archaea. ISME J 14, 597608, doi:10.1038/s41396-019-0552-3 (2020).

18. Benitez-Paez, A. \& Sanz, Y. Multi-locus and long amplicon sequencing approach to study microbial diversity at species level using the MinION portable nanopore sequencer. Gigascience 6, 1-12, doi:10.1093/gigascience/gix043 (2017).

19. Clausen, P., Aarestrup, F. M. \& Lund, O. Rapid and precise alignment of raw reads against redundant databases with KMA. BMC Bioinformatics 19, 307, doi:10.1186/s12859-018-2336-6 (2018). 
20. Marcelino, V. R. et al. CCMetagen: comprehensive and accurate identification of eukaryotes and prokaryotes in metagenomic data. Genome Bio/ 21, 103, doi:10.1186/s13059-020-02014-2 (2020).

21. Te Pas, M. F. W. et al. Sanitary conditions affect the colonic microbiome and the colonic and systemic metabolome of female pigs. Front Vet Sci 7, 585730, doi:10.3389/fvets.2020.585730 (2020).

22. Seshadri, R. et al. Cultivation and sequencing of rumen microbiome members from the Hungate1000 Collection. Nat Biotechno/ 36, 359-367, doi:10.1038/nbt.4110 (2018).

23. Miller, D. A. et al. Complete genome sequence of the cellulose-degrading bacterium Cellulosilyticum lentocellum. J Bacterio/ 193, 2357-2358, doi:10.1128/JB.00239-11 (2011).

24. Callahan, B. J. et al. DADA2: High-resolution sample inference from Illumina amplicon data. Nat Methods 13, 581-583, doi:10.1038/nmeth.3869 (2016).

25. Rosen, M. J., Callahan, B. J., Fisher, D. S. \& Holmes, S. P. Denoising PCR-amplified metagenome data. BMC Bioinformatics 13, 283, doi:10.1186/1471-2105-13-283 (2012).

26. Santos, A., van Aerle, R., Barrientos, L. \& Martinez-Urtaza, J. Computational methods for $16 \mathrm{~S}$ metabarcoding studies using Nanopore sequencing data. Comput Struct Biotechnol J 18, 296-305, doi:10.1016/j.csbj.2020.01.005 (2020).

27. Winand, R. et al. Targeting the $16 \mathrm{~s}$ rrna gene for bacterial identification in complex mixed samples: comparative evaluation of second (Illumina) and third (Oxford Nanopore Technologies) generation sequencing technologies. Int J Mol Sci 21, doi:10.3390/ijms21010298 (2019).

28. Proudman, C. J. et al. Characterisation of the faecal metabolome and microbiome of Thoroughbred racehorses. Equine Vet J 47, 580-586, doi:10.1111/evj.12324 (2015).

29. MM, O. D. et al. The core faecal bacterial microbiome of Irish Thoroughbred racehorses. Lett Appl Microbio/ 57, 492-501, doi:10.1111/lam.12137 (2013).

30. Park, S. C. \& Won, S. Evaluation of 16 S rRNA Databases for Taxonomic assignments using mock community. Genomics Inform 16, e24, doi:10.5808/GI.2018.16.4.e24 (2018).

31. Soergel, D. A., Dey, N., Knight, R. \& Brenner, S. E. Selection of primers for optimal taxonomic classification of environmental 16S rRNA gene sequences. ISME J 6, 1440-1444, doi:10.1038/ismej.2011.208 (2012).

32. Klindworth, A. et al. Evaluation of general 16S ribosomal RNA gene PCR primers for classical and next-generation sequencing-based diversity studies. Nucleic Acids Res 41, e1, doi:10.1093/nar/gks808 (2013).

33. Sipos, R. et al. Effect of primer mismatch, annealing temperature and PCR cycle number on $16 \mathrm{~S}$ rRNA gene-targetting bacterial community analysis. FEMS Microbiol Ecol 60, 341-350, doi:10.1111/j.1574-6941.2007.00283.x (2007).

34. Adam, P. S., Borrel, G., Brochier-Armanet, C. \& Gribaldo, S. The growing tree of Archaea: new perspectives on their diversity, evolution and ecology. ISME J 11, 2407-2425, doi:10.1038/ismej.2017.122 (2017). 
35. Bahram, M., Anslan, S., Hildebrand, F., Bork, P. \& Tedersoo, L. Newly designed 16S rRNA metabarcoding primers amplify diverse and novel archaeal taxa from the environment. Environ Microbiol Rep 11, 487-494, doi:10.1111/1758-2229.12684 (2019).

36. Zeng, Y. H. et al. Long PCR-RFLP of 16S-ITS-23S rRNA genes: a high-resolution molecular tool for bacterial genotyping. J Appl Microbio/ 114, 433-447, doi:10.1111/jam.12057 (2013).

37. Shen, W., Le, S., Li, Y. \& Hu, F. SeqKit: A cross-platform and ultrafast toolkit for FASTA/Q file manipulation. PLoS One 11, e0163962, doi:10.1371/journal.pone.0163962 (2016).

38. Marijon, P., Chikhi, R. \& Varre, J. S. yacrd and fpa: upstream tools for long-read genome assembly. Bioinformatics 36, 3894-3896, doi:10.1093/bioinformatics/btaa262 (2020).

39. Oksanen, J. et al. vegan: Community Ecology Package, <https://CRAN.R-project.org/package=vegan> (2019).

40. Ihaka, R. \& Gentleman, R. R: a language for data analysis and graphics. J Comput Graph Stat 5, 299314 (1996).

41. Wickham, H. ggplot2: Elegant Graphics for Data Analysis, <https://ggplot2.tidyverse.org> (2016).

42. Gu, Z., Eils, R. \& Schlesner, M. Complex heatmaps reveal patterns and correlations in multidimensional genomic data. Bioinformatics 32, 2847-2849, doi:10.1093/bioinformatics/btw313 (2016).

\section{Tables}

Table 1. PERMANOVA of mock community. Model includes PCR target region, PCR polymerase kit, and reference database 


\begin{tabular}{|llllllll|}
\hline $\begin{array}{l}\text { Taxonomic } \\
\text { level }\end{array}$ & Effect & Df & SS & MS & F.Model & R2 & $\operatorname{Pr}(>\mathrm{F})$ \\
\hline Genus level & PCR target region & 1 & 0.20243 & 0.202433 & 8.3050 & 0.51946 & 0.0042 \\
& $\begin{array}{l}\text { PCR polymerase } \\
\text { kit }\end{array}$ & 1 & 0.05565 & 0.055651 & 2.2831 & 0.14281 & 0.0738 \\
& $\begin{array}{l}\text { Reference } \\
\text { Database }\end{array}$ & 1 & 0.03411 & 0.034112 & 1.3995 & 0.08754 & 0.2941 \\
& Residuals & 4 & 0.09750 & 0.024375 & & 0.25019 & \\
\hline Species level & Total & 7 & 0.38970 & & & 1 & \\
\hline & PCR target region & 1 & 0.29283 & 0.292825 & 9.3314 & 0.55341 & 0.0036 \\
\hline & $\begin{array}{l}\text { PCR polymerase } \\
\text { kit }\end{array}$ & 1 & 0.04329 & 0.043285 & 1.3794 & 0.08180 & 0.3081 \\
& $\begin{array}{l}\text { Reference } \\
\text { Database }\end{array}$ & 1 & 0.06750 & 0.067499 & 2.1510 & 0.12757 & 0.1667 \\
\hline & Residuals & 4 & 0.12552 & 0.031381 & & 0.23722 & \\
\hline & Total & 7 & 0.52913 & & & 1 & \\
\hline
\end{tabular}

Df, degrees of freedom; SS, sum of squares; MS, mean squares; $R^{2}$, coefficient of determination.

Table 2. Detailed information of sequence reads with unlinked rRNA genes

\begin{tabular}{|clll|}
\hline Horse & Sequence id & $\begin{array}{l}\text { Read mapping in KMA software } \\
\text { with ncbi 202006 DB }\end{array}$ & $\begin{array}{l}\text { Taxonomic assignment in } \\
\text { CCMetagen software }\end{array}$ \\
\hline A & $\begin{array}{l}\text { 22eb24f6-ab8c-4935- } \\
\text { 9581-c65b2a9d1afa }\end{array}$ & $\begin{array}{l}\text { 642492|NC_015275.1 } \\
\text { Cellulosilyticum lentocellum DSM } \\
\text { 5427 }\end{array}$ & Clostridiales \\
\hline & $\begin{array}{l}\text { 9cfd48d6-1be8-4f1e- } \\
\text { b72a-fef9e5495205 }\end{array}$ & $\begin{array}{l}\text { 1527|NZ_OAOG01000091.1 } \\
\text { Anaerocolumna aminovalerica }\end{array}$ & Lachnospiraceae \\
\hline S & $\begin{array}{l}\text { dd8fdda9-49f6-4d6a- } \\
\text { 9c09-ccee93bb6a59 }\end{array}$ & $\begin{array}{l}\text { 642492|NC_015275.1 C. } \\
\text { lentocellum DSM 5427 }\end{array}$ & Firmicutes \\
\hline L & $\begin{array}{l}\text { 15fc239f-2794-40e4- } \\
\text { acaa-1a8e1d31cc07 }\end{array}$ & $\begin{array}{l}\text { 1294025|NZ_BBCG01000153.1 C. } \\
\text { ruminicola JCM 14822 }\end{array}$ & Lachnospiraceae \\
\hline & $\begin{array}{l}\text { 45df32f4-6390-487a- } \\
\text { 99d4-75ef1b78e773 }\end{array}$ & $\begin{array}{l}\text { 642492|NC_015275.1 C. } \\
\text { lentocellum DSM 5427 }\end{array}$ & Clostridiales \\
\hline & $\begin{array}{l}\text { 715b9279-6443-4a46- } \\
\text { a258-2972c2d64712 }\end{array}$ & $\begin{array}{l}\text { 1294025|NZ_BBCG01000153.1 C. } \\
\text { ruminicola JCM 14822 }\end{array}$ & Lachnospiraceae \\
\hline
\end{tabular}

\section{Figures}


a) Genus level

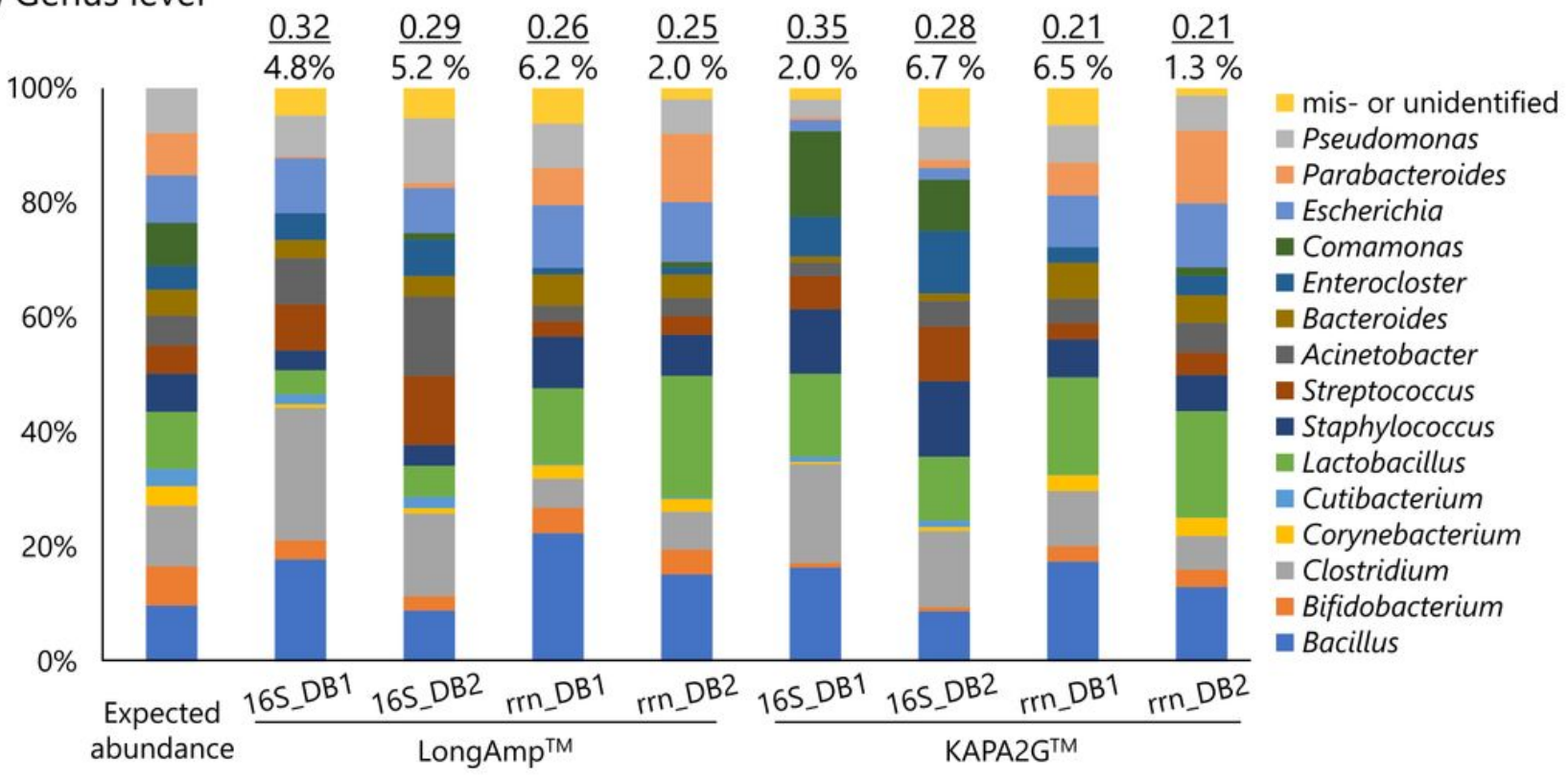

b) Species level

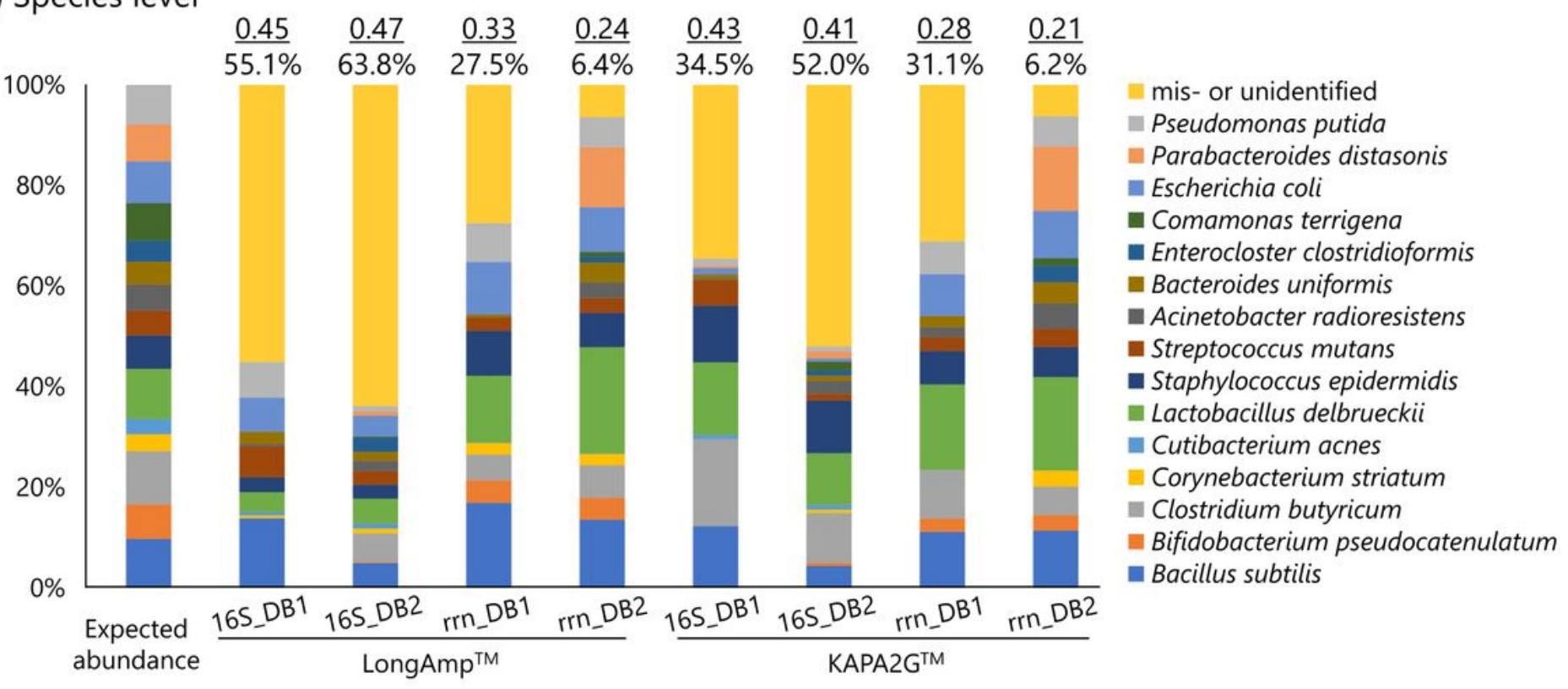

Figure 1

Relative abundance of mock community at (a) genus and (b) species levels. Indices of dissimilarities between the expected abundance and each sequencing condition (underlined) and the percentages of mis- or unidentified sequences are shown above each bar graph. 16S: full-length 16S rRNA gene amplicon sequencing. rrn: rRNA operon amplicon sequencing. DB1: rrn DB18. DB2: ncbi_202006 DB. 


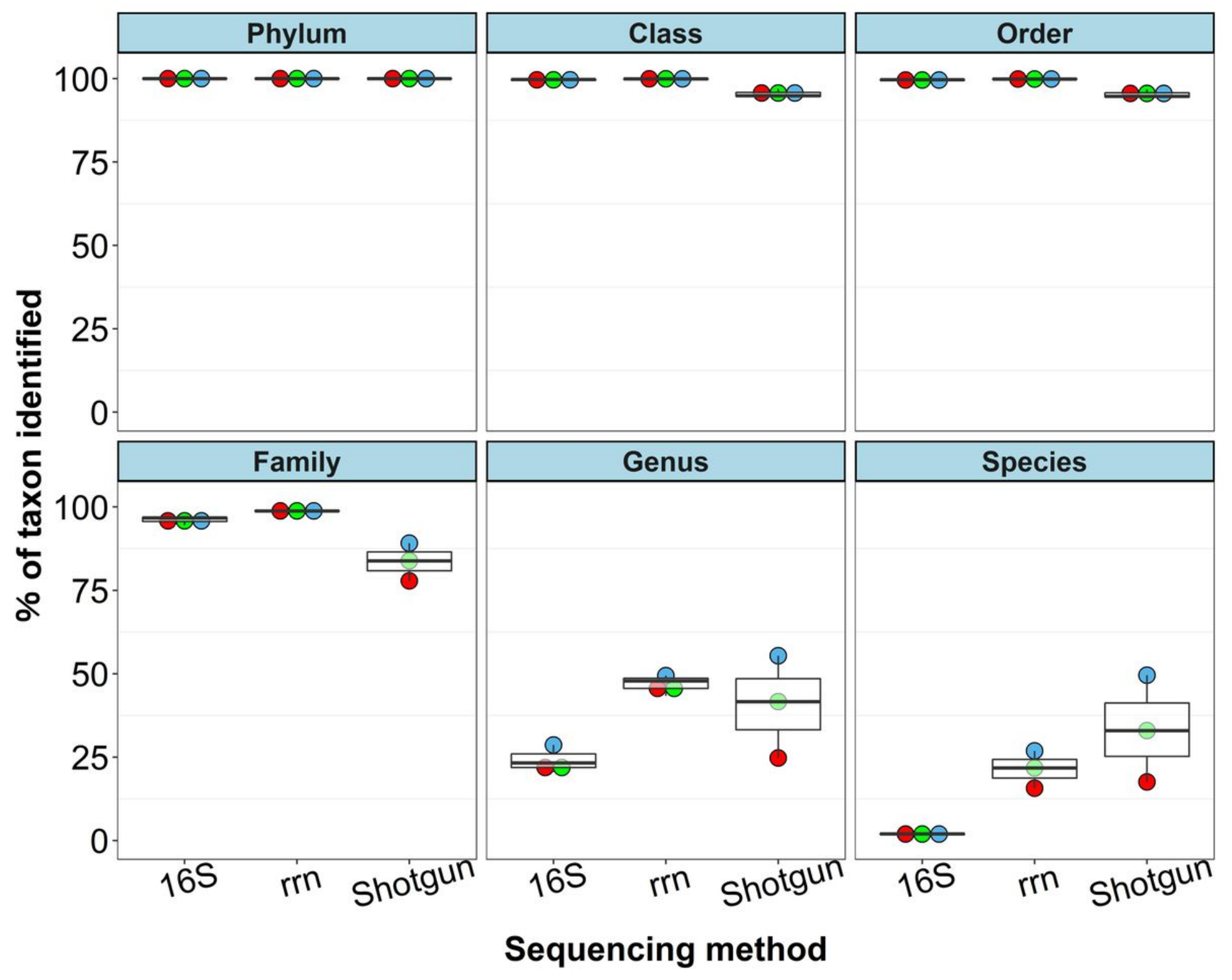

Figure 2

Rates of reads assigned to each taxon from phylum to species level. Each point shows each horse: red, horse A; blue, horse S; green, horse L. 16S: full-length 16S rRNA gene amplicon sequencing. rrn: rRNA operon amplicon sequencing. Shotgun: shotgun sequencing. 


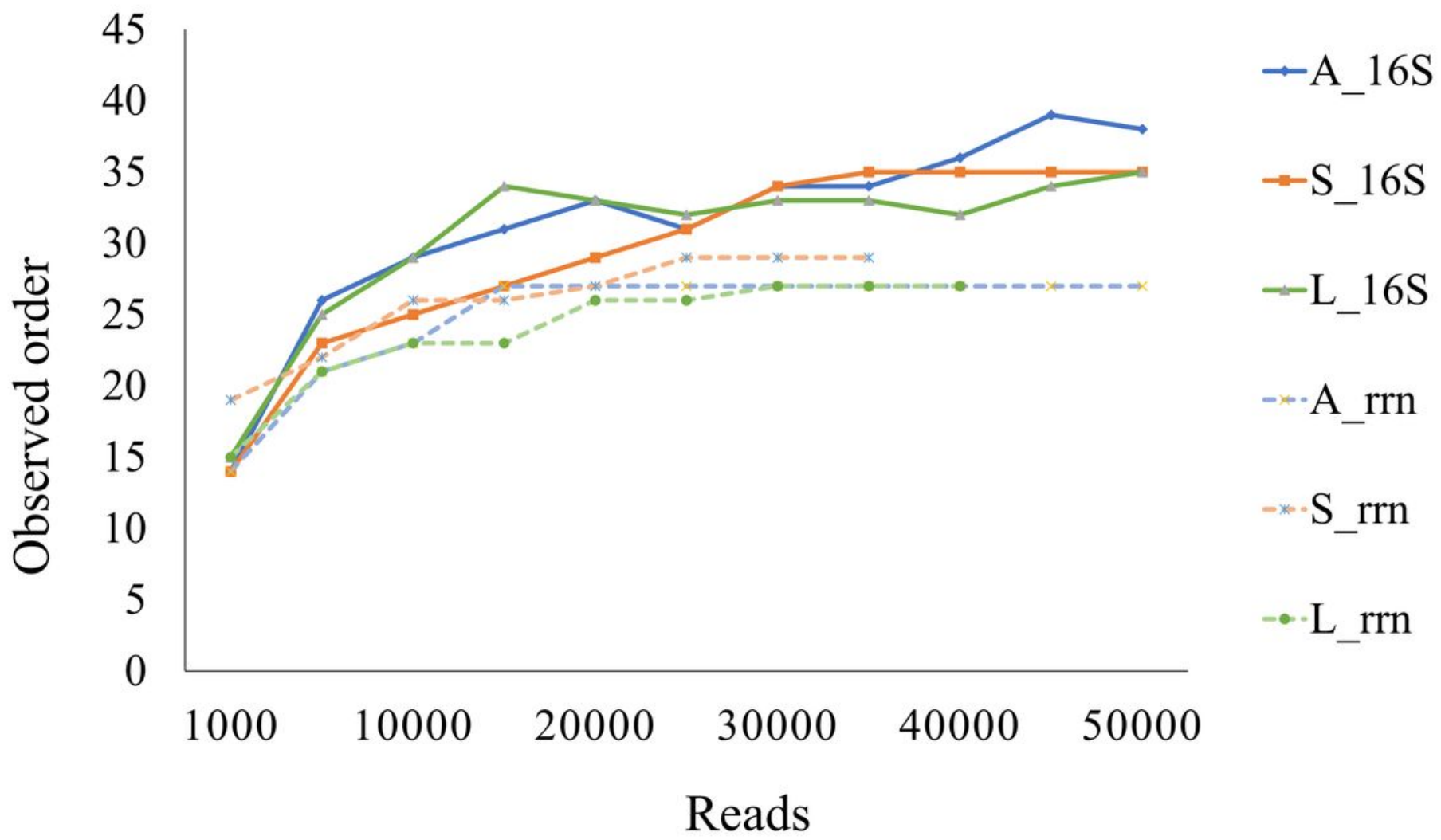

Figure 3

Numbers of observed orders against numbers of sequence reads. Three equine fecal samples $(A, S$, and L) were subjected to full-length 16S rRNA gene amplicon sequencing (16S) and rRNA operon amplicon sequencing (rrn). 


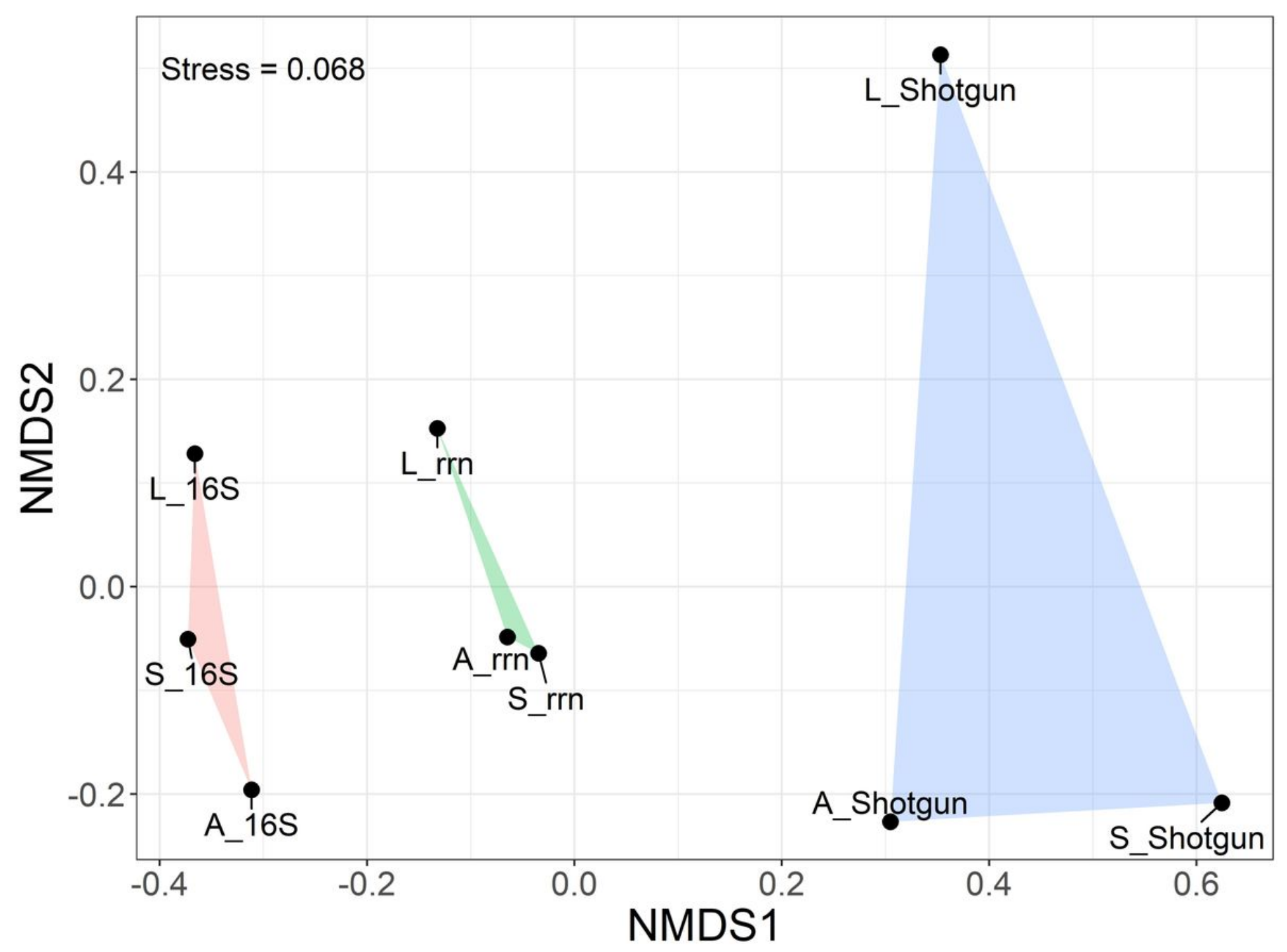

Sequencing method $\square$ 16S $\square$ rn $\square$ Shotgun

\section{Figure 4}

NMDS analysis of the microbial communities in equine fecal samples. Three equine fecal samples (A, $S$, and $L$ ) formed significant groups by sequencing method (PERMANOVA: $P=0.0037, R 2=0.74328$ ). $16 \mathrm{~S}$ : full-length 16S rRNA gene amplicon sequencing. rrn: rRNA operon amplicon sequencing. Shotgun: shotgun sequencing. 


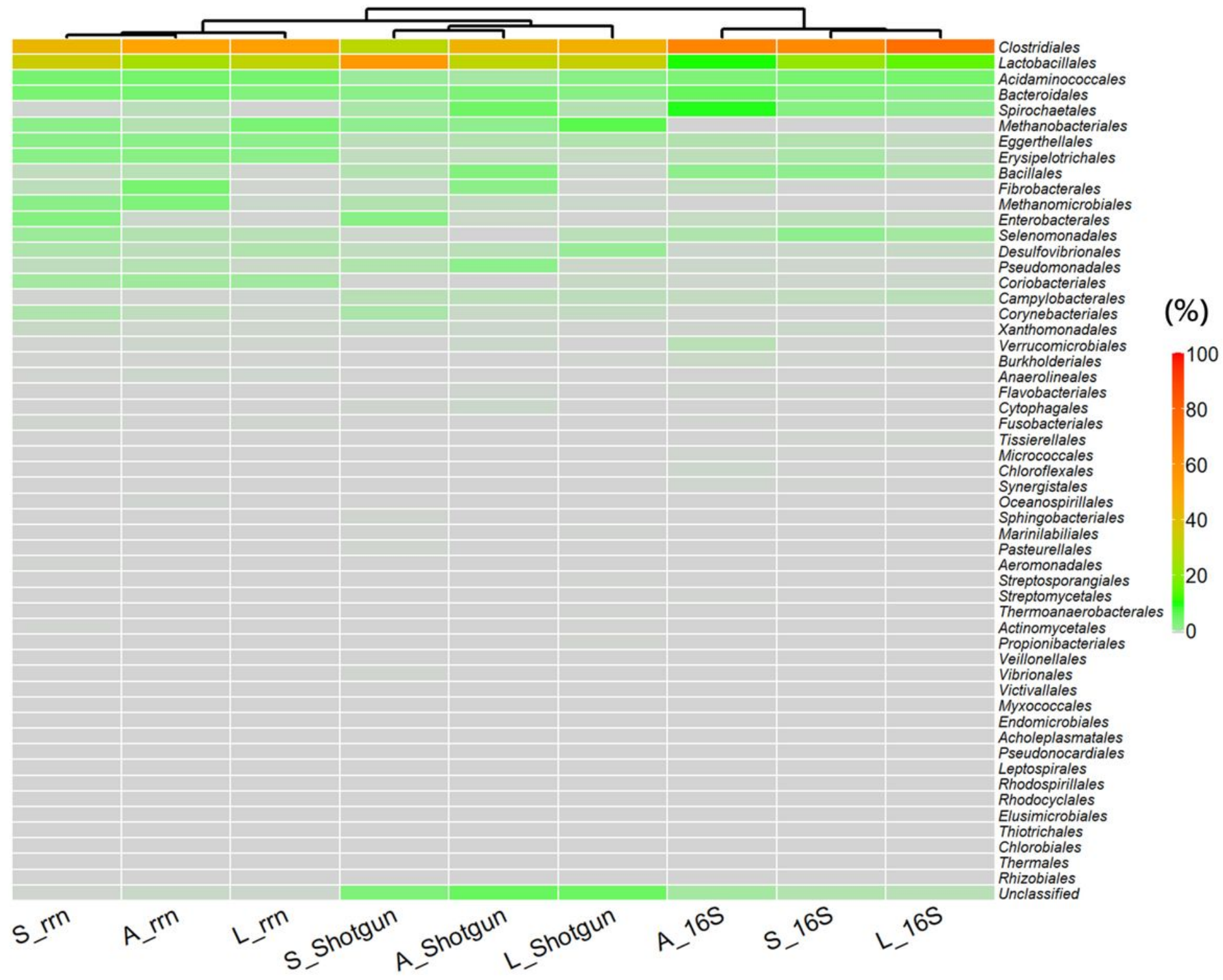

Figure 5

Heat map of each order assigned and hierarchical clustering of sample types. The hierarchical clustering shows that the samples from the amplicon sequencing with rRNA operon are grouped with those from shotgun sequencing. Sample types are shown as in Fig. 4. 

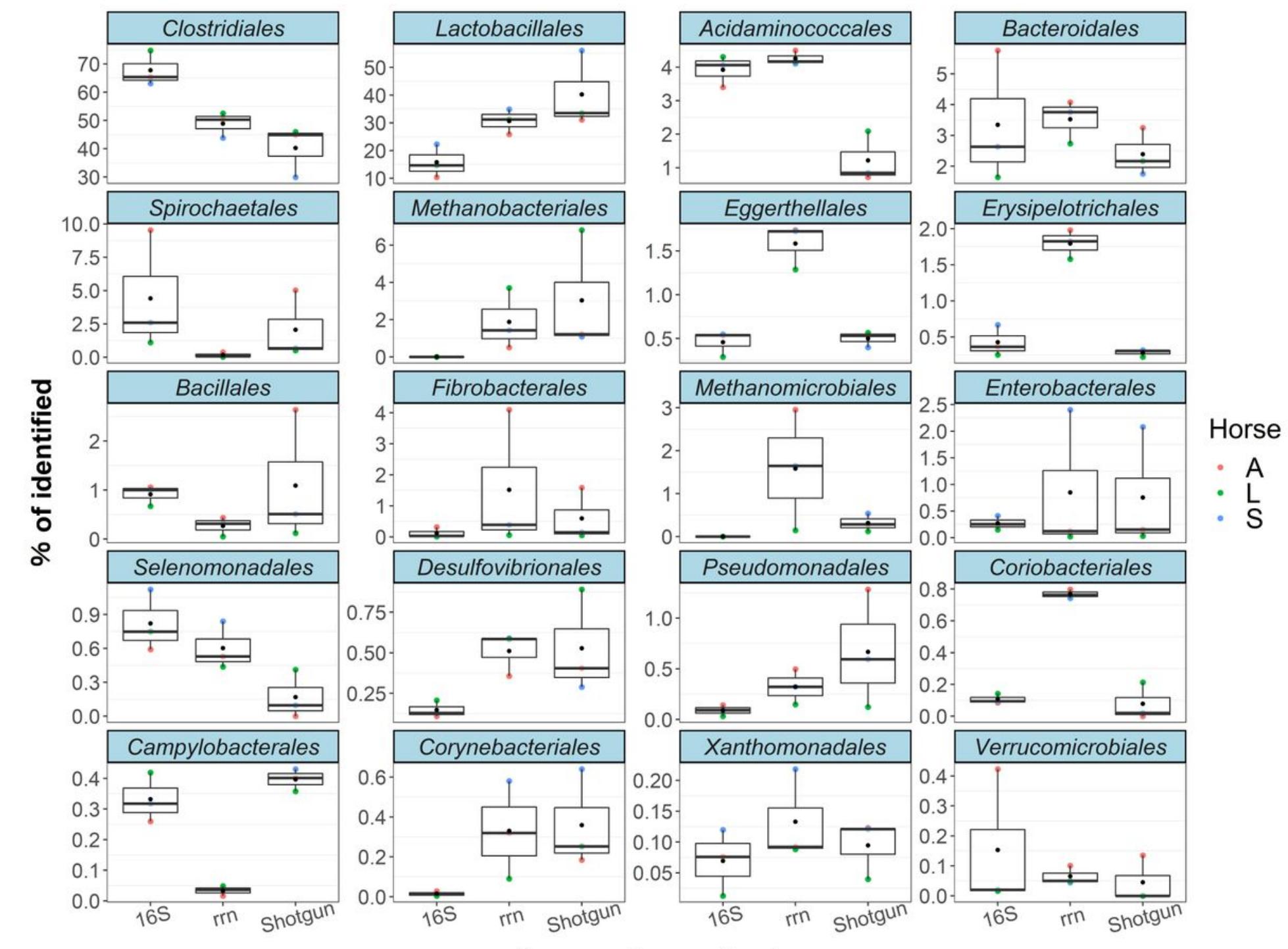

\section{Figure 6}

Boxplots of the top 20 orders with the highest average percentages. Sequencing methods are shown as in Fig. 2. Average values of the three horses were shown as black dot.

\section{Supplementary Files}

This is a list of supplementary files associated with this preprint. Click to download.

- SupplementaryTable1.xlsx

- SupplementaryTable2.xIsx

- SupplementaryTable4.xlsx

- SupplementaryTable5.xlsx

- SupplementaryTable6.xIsx

- SupplementaryTable7.xlsx 
- Titleforthesupplementarymaterial.docx 